\title{
Structural Health Monitoring Exploiting MIMO Ultrasonic Sensing and Group Sparse Bayesian Learning
}

\author{
Qisong Wu, Yimin D. Zhang, Moeness G. Amin, Andrew Golato, Fauzia Ahmad, and Sridhar Santhanam \\ Center for Advanced Communications, Villanova University, Villanova, PA 19085, USA
}

\begin{abstract}
In this paper, we propose the exploitation of sparse Bayesian learning in multiple-input multiple-output (MIMO) systems to account for the multi-dimensional group sparse nature of extended defects in guided ultrasonic wave based structural health monitoring. The multi-dimensional group sparsity in the underlying reconstruction problems arises due to the clustered spatial occupancy of extended defects and the multiple-aspect MIMO observations. Sparse Bayesian learning techniques have been shown to provide robustness for high-resolution signal reconstruction due to their insensitivity to dictionary coherence and have the flexibility of effective exploitation of the signal structure. The superiority of the proposed technique over the state-ofthe-art sparse signal reconstruction techniques is demonstrated through simulations and preliminary experiments.
\end{abstract}

Index Terms-Structure health monitoring, multiple-input multiple-output (MIMO), group sparsity, Bayesian compressive sensing

\section{INTRODUCTION}

Structural health monitoring (SHM) is a collection of strategies and tools for automated inspection and examination of critical structures, such as bridges and aircrafts, in order to assess their safety and viability $[1,2]$. These strategies rely on the use of sensors integrated into the structures, with the sensors continuously monitoring the structures for the existence of defects and other anomalies. Ultrasonic guided waves are emerging as a very effective tool to perform SHM for certain mechanical structures $[3,4]$. For structures that resemble plates and shells, Lamb waves are the preferred modality for detecting and classifying defects $[5,6]$. Lamb waves are ultrasonic guided waves that propagate through plates with traction free surfaces [7]. These waves can propagate large distances with little attenuation. Lamb waves also interact strongly with defects, such as cracks, holes, gouges, and corrosion [8-10]. Hence, broad areas of structures can be efficiently and economically examined for the presence of defects with a limited set of transducers.

Although Lamb wave based SHM is very promising, there are many challenges that add complexities [11]. One principal challenge is that Lamb wave propagation is multimodal in nature. When a guided wave is instigated in a plate at a given frequency, it can propagate as multiple modes, each with a different wave number. These multiple modes can be separated into symmetric (S) and anti-symmetric (A) modes. The total number of symmetric and anti-symmetric modes present is determined by the frequency of operation. At low frequencies, only the fundamental symmetric $\left(\mathrm{S}_{0}\right)$ and anti-symmetric

This work was supported in part by the National Science Foundation (NSF) under grant number IIP-0917690.
$\left(\mathrm{A}_{0}\right)$ modes co-exist. To overcome the complexity caused by multiple modes, an operating frequency is chosen wherein the dominant mode of propagation is either the fundamental $\mathrm{A}_{0}$ mode or the $\mathrm{S}_{0}$ mode. Another challenge is that all modes are dispersive since the phase and group velocities of each individual mode are a function of frequency. This challenge is overcome by modeling the dispersion phenomenon accurately. Finally, boundary scattering can add complexity in the received signal. This too can be ameliorated by using background subtraction [12].

Several techniques have been developed for imaging defects in two dimensional plates using Lamb waves. Some success has been achieved with methods, such as delay-and-sum beamforming $[13,14]$. However, there are issues with these methods including poor resolution and false defects. Sparse reconstruction approaches have recently been successfully applied to Lamb wave based SHM. Levine et al. [15, 16] first cast the defect imaging problem in Lamb wave based SHM within the sparse reconstruction framework. However, the defects examined in their work were essentially point defects and not extended defects.

In this paper, we examine the problem of locating extended defects in an aluminum plate under multiple-input multiple-output (MIMO) sensing with a limited number of distributed transducers using multi-dimensional group sparse Bayesian learning techniques [17]. The underlying problem exhibits a multi-dimensional group sparse structure because i) the defects have an extended spatial occupancy that is clustered in the image domain, and ii) the MIMO sensing methodology results in multi-aspect observations of the same defect. Sparse Bayesian learning techniques are known to provide robustness for high-resolution signal reconstruction due to their insensitivity to dictionary coherence and provide the flexibility of effective exploitation of the signal structure $[18,19]$. As such, the proposed approach leads to an effective high-resolution imaging methodology. The superiority of the proposed technique over the state-of-the-art sparse signal reconstruction techniques is demonstrated through simulations and experiments of a thin aluminum plate with an extended defect resembling a crack.

Notations. We use lower-case (upper-case) bold characters to denote vectors (matrices). $\mathcal{F}_{x}$ and $\mathcal{F}_{x}^{-1}$ respectively represent the Fourier Transform and inverse FT with respect to $x$. In particular, $\mathbf{I}_{N}$ denotes the $N \times N$ identity matrix. $(\cdot)^{T}$ and $(\cdot)^{*}$ respectively denote the transpose and complex conjugates of a matrix or vector, $p(\cdot)$ denotes the probability density function (pdf), and $\mathcal{C N}(x \mid a, b)$ implies that random variable $x$ follows a complex Gaussian distribution with mean $a$ and variance $b$. 
Furthermore, $\delta(x)$ is the Dirac delta function of $x$, and ' $\circ$ ' denotes element-wise (Hadamard) multiplication.

\section{SignAl MODEL}

We consider $J$ spatially distributed piezoelectric transducers attached to the surface of a plate structure. The transducers transmit in a sequential manner, i.e., when one transducer transmits, all the other transducers act as receivers. This makes the waveforms orthogonal by virtue of time multiplexing. A total of $L=J(J-1) / 2$ unique transmitter-receiver pairs are used for interrogating the region of interest. We divide the region of interest into a uniform grid of $M$ points or pixels, where each pixel represents a potential defect location. In general, the number of defects is typically small compared to $M$. Let the transmitter of the $l$ th pair, located at $\mathbf{t}_{l}$, be excited by a waveform $h(t)$ whose center frequency is chosen such that the dominant propagating mode in the plate is the $\mathrm{A}_{0}$ mode. The received signal at the receiver of the $l$ th pair, located at $\mathbf{r}_{l}$, can be expressed as,

$$
\begin{aligned}
y_{l}(t) & =\sum_{m=0}^{M-1} w_{i l}\left(\frac{a}{\left\|\mathbf{t}_{l}-\mathbf{s}_{i}\right\|_{2}}\right)^{0.5}\left(\frac{a}{\left\|\mathbf{r}_{l}-\mathbf{s}_{i}\right\|_{2}}\right)^{0.5} \\
& \times \mathcal{F}^{-1}\left\{H(f) \exp \left[j 2 \pi f \frac{\left\|\mathbf{t}_{l}-\mathbf{s}_{i}\right\|_{2}+\left\|\mathbf{r}_{l}-\mathbf{s}_{i}\right\|_{2}}{c_{\mathrm{A}_{0}}(f)}\right]\right\},
\end{aligned}
$$

where $H(f)$ is the Fourier transform of $h(t), c_{\mathrm{A}_{0}}(f)$ is the frequency-dependent phase velocity of the $\mathrm{A}_{0}$ mode, $\mathrm{s}_{i}$ is the $i$ th pixel location, and $a$ is an arbitrary constant with dimensions of length. The inverse square root dependence on $\left\|\mathbf{t}_{l}-\mathbf{s}_{i}\right\|_{2}$ and $\left\|\mathbf{r}_{l}-\mathbf{s}_{i}\right\|_{2}$ accounts for the attenuation due to the geometrical spreading of the wavefront on transmit and receive paths to the $i$ th pixel. The parameter $w_{i l}$ is the reflectivity of the $i$ th pixel corresponding to the $l$ th transmitter-receiver pair. If no defect is present at the $i$ th pixel, then $w_{i l}=0, \forall l$. As such, the reflectivity vector of $\mathbf{w}_{l}$ can be considered as sparse due to much less defects in the region of interest.

An equivalent matrix-vector representation of the signals $y_{l}(t), l=[0,1, \cdots, L-1]$, is obtained as follows. The $l$ th received signal is sampled at times $t_{k}, k=[0,1, \cdots, K-1]$ to obtain a $K \times 1$ vector $\mathbf{y}_{l}=\left[y_{l}\left(t_{0}\right), \ldots, y_{l}\left(t_{K-1}\right)\right]^{T}$. Let $\mathbf{w}_{l}=\left[w_{1 l}, \ldots, w_{(M-1) l}\right]^{T}$ be the $M \times 1$ scene reflectivity vector corresponding to the $l$ th transmitter-receiver pair. Then, using Eq. (1), we obtain the linear relation

$$
\mathbf{y}_{l}=\boldsymbol{\Phi}_{l} \mathbf{w}_{l}+\epsilon_{l},
$$

for $l \in[0,1, \cdots, L-1]$, where the $(k, i)$ th element of the $K \times M$ sensing matrix $\boldsymbol{\Phi}_{l}$ is given by

$$
\begin{aligned}
& {\left[\boldsymbol{\Phi}_{l}\right]_{k, i}=\left(\frac{a}{\left\|\mathbf{t}_{l}-\mathbf{s}_{i}\right\|_{2}}\right)^{0.5}\left(\frac{a}{\left\|\mathbf{r}_{l}-\mathbf{s}_{i}\right\|_{2}}\right)^{0.5}} \\
& \times \mathcal{F}^{-1}\left\{H(f) \exp \left[j 2 \pi f \frac{\left\|\mathbf{t}_{l}-\mathbf{s}_{i}\right\|_{2}+\left\|\mathbf{r}_{l}-\mathbf{s}_{i}\right\|_{2}}{c_{\mathrm{A}_{0}}(f)}\right]\right\}_{t=t_{k}},
\end{aligned}
$$

and an additive noise is considered in Eq. (2). It should be noted that the defect share the group sparsity across the $L$ transmitter-receiver pairs, i.e., the supports of the defect are approximately identical for these pairs, which implies that the nonzero entries of $\mathbf{w}_{l}$ lie in the same positions across different values of $l$, whereas their reflectivities are different.

\section{Structure Bayesian Compressive Sensing METHOD}

To solve the group sparse reconstruction problem with $L$ transmitter-receiver pairs as described in Eq. (2), we exploit the sparse Bayesian learning techniques which are known to provide robustness for high-resolution signal reconstruction due to their insensitivity to dictionary coherence and provide the flexibility of effective exploitation of the signal structure. In particular, we place the following spike-and-slab prior on the reflectivity vector $\mathbf{w}_{l}$ to enforce group sparsity across all pairs [17, 20],

$$
p\left(\mathbf{w}_{l} \mid \boldsymbol{\pi}, \boldsymbol{\beta}\right)=\prod_{i=1}^{M}\left[\left(1-\pi_{i}\right) \delta\left(w_{i l}\right)+\pi_{i} \mathcal{C N}\left(w_{i l} \mid 0, \beta_{i}^{-1}\right)\right]
$$

where $\beta_{i}$ is the precision (reciprocal of the variance) of the Gaussian distribution and $\pi_{i}$ is the prior probability of a nonzero element in the $i$ th pixel. It can be observed in the above expression that a large weight $\pi_{i}$ corresponds to a high probability that the entry takes a nonzero value, whereas a small $\pi_{i}$ tends to generate a zero entry. It is also important to mention that the prior probability $\pi_{i}$ defined in the $i$ th pixel is shared across all the transmitter-receiver pairs, thereby encouraging group sparsity in the underlying hierarchical Bayesian compressive sensing (BCS) framework.

To make the inference analytical, a simple reparameterization of the spike-and-slab prior is employed. Two latent random variables, $\boldsymbol{\theta}_{l}$ and $\mathbf{z}$, which respectively follow complexvalued Gaussian and Bernoulli distributions, are introduced, and their element-wise product $\boldsymbol{\theta}_{l} \circ \mathbf{z}$ forms a new random vector that follows the pdf in (4), i.e.,

$$
p\left(\boldsymbol{\theta}_{l}, \mathbf{z}\right)=\prod_{i=1}^{M}\left[\mathcal{C N}\left(\theta_{i l} \mid 0, \beta_{i}^{-1}\right)\right]^{z_{i}} \operatorname{Bern}\left(z_{i} \mid \pi_{i}\right) .
$$

In this case, the group sparsity is characterized by the same $z_{i}$, which takes a binary value representing whether a pixel is zero valued, for the $i$ th pixels across the $L$ pairs. On the other hand, the reflectivities $\boldsymbol{\theta}_{i}=\left[\theta_{i 1}, \cdots, \theta_{i L}\right]$, in general, take different values for each transmitter-receiver pair.

To acquire the trackable posterior of $\beta_{i}$, we place a Gamma prior, which is conjugate to the Gaussian distribution, on $\beta_{i}$, i.e., $\beta_{i} \sim \operatorname{Gamma}(a, b), i \in[1, \cdots, M]$, where $a$ and $b$ are hyper-parameters. A Gaussian prior is placed on the additive noise as $\boldsymbol{\epsilon}_{l} \sim \mathcal{C N}\left(\boldsymbol{\epsilon}_{l} \mid \mathbf{0}, \alpha_{l}^{-1} \mathbf{I}_{N}\right)$. In a similar manner, a Gamma prior is placed on $\alpha_{l}$ to acquire an analytical posterior distribution, i.e., $\alpha_{l} \sim \operatorname{Gamma}(c, d), l \in[1, \cdots, L]$, where $c$ and $d$ are hyper-parameters.

In the underlying application, the defect is likely to take an continuous two-dimensional (2-D) structure. To incorporate such dependency between neighboring pixels, three kinds of sparsity patterns are introduced. As depicted in Fig. 1, we categorize the relationship into three different clustering patterns, i.e., Pattern 0 ("strong rejection"), Pattern 1 ("weak rejection"), and Pattern 2 ("strong acceptance"). We define two 


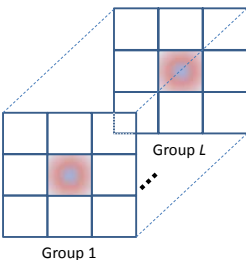

(a)

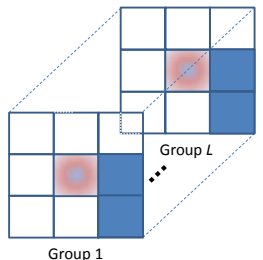

(b)

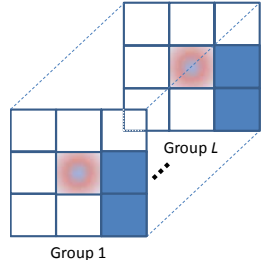

(c)
Fig. 1: Examples of clustering patterns for a 2-D image. (a) Strong rejection example with $\kappa_{i}=0$; (b) Weak rejection example with $\kappa_{i}=2$ and $\eta_{i} \neq 1$ case; and (c) Strong acceptance example with $\eta_{i}=1$.

variables $\kappa_{i}=\sum_{n=1}^{8} \tilde{z}_{n}^{(i)}$ and $\eta_{i}=\sum_{n=1}^{4} \tilde{z}_{n}^{(i)} \tilde{z}_{9-n}^{(i)}$, which respectively represent the number of nonzero neighboring entries and the number of nonzero diagonal pairs, where $\tilde{z}_{n}^{(i)}$ is the $n$th neighboring pixel with respect to the $i$ th pixel under test [21]. To facilitate the inference, a Beta prior, which is conjugate to the Bernoulli distribution, with different sets of parameters $\left\{e_{i}, f_{i}\right\}_{i=0}^{2}$, is placed on weights $\pi_{i}$ according to the clustering patterns.

With respect to the cluster pattern in the 1-D case in [17], it is straightforward to assign three-level cluster patterns according to the neighboring pixel number. However, in the underly SHM where defect exhibits a 2-D cluster structure with a higher number of neighboring pixels. Following the same pattern, a stepped function is introduced to assign corresponding pattern based on each $\kappa_{i}$.

Strong rejection [Pattern 0]: This pattern is adopted when all neighboring pixels are zero, i.e., $\kappa_{i}=0$, as illustrated in Fig. 1(a). In this case, the $i$ th pixel under test would be zero with a high probability, since the defect pixel is unlikely to be isolated in the underlying defect imaging. We assume $e_{0}<f_{0}$ in prior $\operatorname{Beta}\left(e_{0}, f_{0}\right)$ to encourage a small value of $\pi_{i}$ so as to reject this pixel.

Weak rejection [Pattern 1]: When the number of nonzero neighboring pixels $\kappa_{i}>0$ and the nonzero diagonal pair $\eta_{i} \neq$ 1 , the probability that the $i$ th pixel under test takes zero value is fair, and $e_{1}=f_{1}$ is used in the prior $\operatorname{Beta}\left(e_{1}, f_{1}\right)$ to exert noninformative prior on $\pi_{l}$. Fig. 1(b) shows one of such examples where two nonzero neighboring pixels do not form a diagonal pair.

Strong acceptance [Pattern 2]: When the number of nonzero diagonal pair $\eta_{i}$ is 1 , the $i$ th pixel under test would be nonzero with a high probability so that the defect continuity can be enforced. In this case, $e_{2}>f_{2}$ in the prior $\operatorname{Beta}\left(e_{2}, f_{2}\right)$ is assumed to encourage a large value of $\pi_{i}$ to accept this pixel. Fig. 1(c) shows one of examples with a pair of nonzero diagonal pixels.

By exploiting the hieratical spike-and-slab prior for the group sparsity across $L$ transmitter-receiver pairs and the cluster pattern for the underlying structure of defect, we acquire generative model for the clustered multi-task BCS algorithm. A Gibbs sampler is employed to carry out the Bayesian inference.

Utilizing the conjugate properties, we analytically acquire the respective posterior distributions for each random variable $\{\mathbf{z}, \boldsymbol{\theta}, \boldsymbol{\pi}, \boldsymbol{\alpha}, \boldsymbol{\beta}\}$. The posterior probability of $z_{i}=1$ given other variables is acquired analytically by,

$$
p\left(z_{i}=1 \mid-\right)=\left(1+e^{-u}\right)^{-1},
$$

with

$$
\begin{aligned}
u= & \frac{1}{2} \sum_{l=1}^{L}\left(\log \beta_{i}-\log \sigma_{i l}+\sigma_{i l}^{-1} \alpha_{l}^{2} \mathbf{y}_{\backslash i l}^{H} \boldsymbol{\phi}_{i l} \boldsymbol{\phi}_{i l}^{H} \mathbf{y} \backslash i l\right) \\
& \quad+\log \pi_{i}-\log \left(1-\pi_{i}\right), \\
\sigma_{i l}= & \left(\alpha_{l} \boldsymbol{\phi}_{i l}^{H} \boldsymbol{\phi}_{i l}+\beta_{i}\right)^{-1},
\end{aligned}
$$

where $\mathbf{y}_{\backslash i l}=\mathbf{y}_{l}-\sum_{k \neq i} \phi_{k l} z_{k} \theta_{k l}$, and $\phi_{i l}$ is the $i$ th column of $\boldsymbol{\Phi}_{l}$.

For $z_{i}=1$, the posterior distribution of $\theta_{i l}$ can be given by,

$$
p\left(\theta_{i l} \mid-\right)=\mathcal{C N}\left(\theta_{i l} \mid \sigma_{i l}^{-1} \alpha_{l} \boldsymbol{\phi}_{i l}^{H} \mathbf{y} \backslash i l_{i l}, \sigma_{i l}\right) .
$$

For $z_{i}=0$, the value of variable $\theta_{i l}$ is drawn from its prior,

$$
p\left(\theta_{i l} \mid-\right)=\mathcal{C N}\left(\theta_{i l} \mid 0, \beta_{i}^{-1}\right) .
$$

Once both $\boldsymbol{\theta}_{l}$ and $\mathbf{z}$ are acquired, $\mathbf{w}_{l}=\boldsymbol{\theta}_{l} \circ \mathbf{z}$ is exactly the estimated reflectivity vector in the $l$ th transmitter-receiver pair. The updated formulations for other random variables $\{\boldsymbol{\pi}, \boldsymbol{\alpha}, \boldsymbol{\beta}\}$ are provided in [17].

\section{Simulations AND EXPERIMENTS}

In this section,both simulated and real data experiments are performed to validate the effectiveness of proposed approach.

\section{A. Simulations}

The simulated setup is shown in Fig. 2, and simulation arrangements are as follows: the square region of interest consists of $31 \times 31$ pixels with side dimensions extending from $-150 \mathrm{~mm}$ to $150 \mathrm{~mm}\left(300 \times 300 \mathrm{~mm}^{2}\right)$. Five sensors are located on a circle with a radius of $250 \mathrm{~mm}$. The two transducers, respectively located at the 7 and 9 o'clock positions, are chosen as transmitters, and the transducer located at the 12 o'clock position is designated as a receiver. The waveforms in the sensing matrix $\boldsymbol{\Phi}_{l}$, generated by Eq. (3), are provided in Fig. 3(a), and Fig. 3(b) shows the enlarged plot for the first 31 pixels. Since the measurement noise can be substantially reduced by averaging over multiple measurements, we do not take into consideration the effect of noise. For comparison, several state-of-the-art algorithms, such as back-projection (BP), block orthogonal matching pursuit (BOMP) [22], and multi-task Bayesian compressive sensing (MT-BCS) algorithm [18], are also applied to the simulated data.

A continuous crack consisting of 13 pixels is assumed around the center of the circle, as shown in Fig. 4(a), and the reflectivities follow a zero-mean complex Gaussian distribution. The corresponding received waveforms are depicted in Fig. 4(b). It is evident that the low-resolution reconstructed result obtained from BP algorithm, shown in Fig. 5(a), is of poor quality due to the lack of a sufficient number of measurements. Although BOMP based on the greedy algorithm has the capability of acquiring high-resolution images, it fails to accurately localize the extended defect in the underlying case because of the high coherence between the waveforms in the 


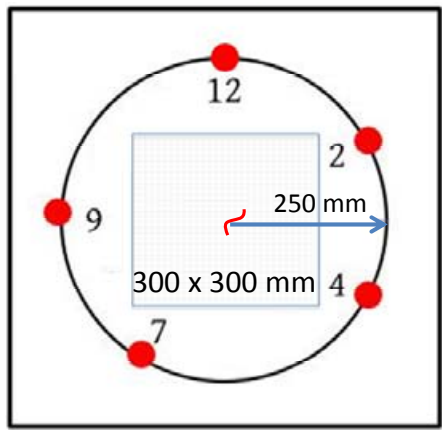

Fig. 2: Simulation setup

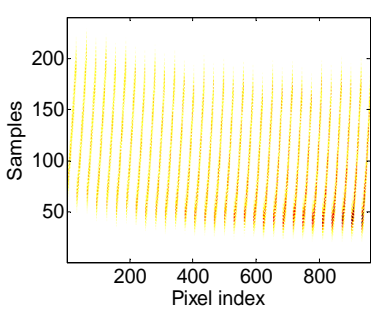

(a)

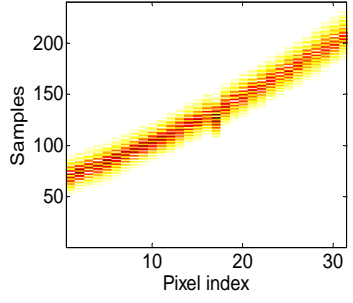

(b)
Fig. 3: Waveforms in sensing matrix. (a) Waveforms for all pixels. (b) Amplified waveforms for the first 31 pixels.

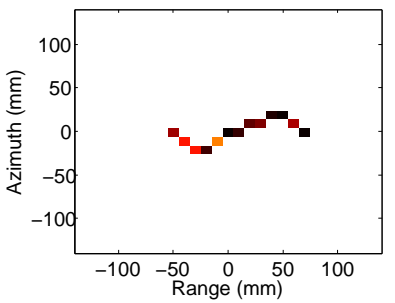

(a)

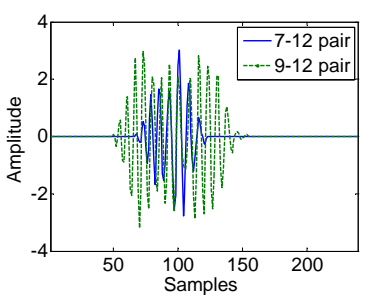

(b)
Fig. 4: Simulated scenario and measurement waveforms. (a) Continuous cracks. (b) Real measurement waveforms.

sensing matrix, as evident from Fig. 3. Since BCS algorithms are capable of reconstructing sparse signals under highly coherent sensing matrices, Figs. 5(c) and 5(d), respectively, show significantly improved results. It is observed, however, that the proposed algorithm preserves the continuous cracks and suppresses the isolated pixels by exploiting both group sparsity and cluster structure, and thus has much cleaner background in Fig. 5(d), compared to that for MT-BCS in Fig. 5(c).

\section{B. Experiments}

The experiment is conducted with a $1.22 \mathrm{~m}$ square aluminum plate of thickness $3.12 \mathrm{~mm}$. An array of five PZT transducers (APC International), $0.22 \mathrm{~mm}$ thick with a $10 \mathrm{~mm}$ diameter, are arranged around a circle of radius $250 \mathrm{~mm}$ at the $12,2,4,7$, and 9 oclock positions, identical to the arrangement assumed in the simulations (Fig. 2). A Hanning-windowed, five-cycle burst of a $150 \mathrm{kHz}$ sinusoidal signal, generated using a National Instruments (NI) PXI 5142 Arbitrary Waveform

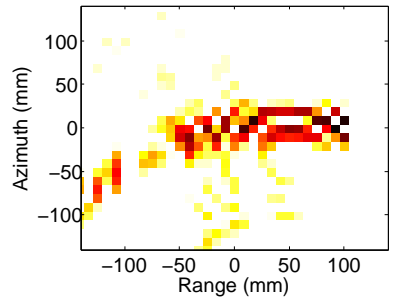

(a)

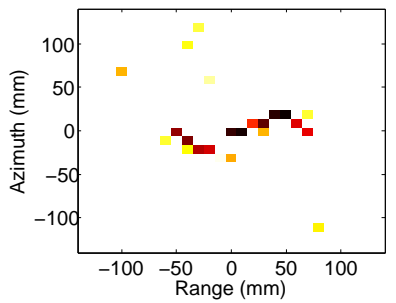

(c)

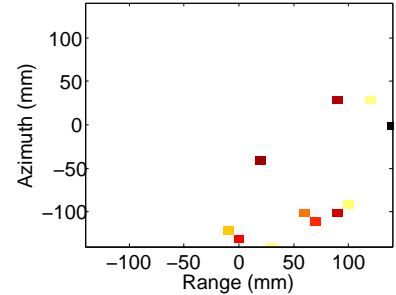

(b)

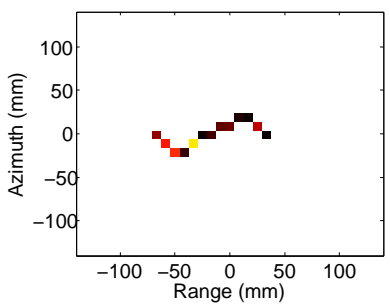

(d)
Fig. 5: Reconstructed results. (a) Result obtained from BP. (b) Result obtained from OMP. (c) Result obtained from MT-BCS. (d) Result obtained from the proposed approach.

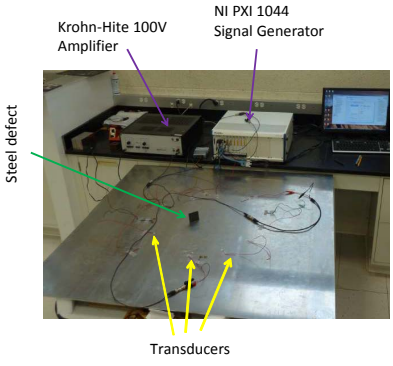

(a)

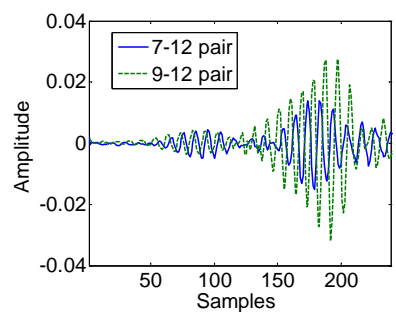

(b)
Fig. 6: SHM experiment setup. (a) Experiment scene. (b) Experimental measurement waveforms.

Generator, is used to excite the transmitting transducers. In order to reduce noise, measurements are averaged over 5000 collections in LabView via an NI PXI 5105 Digitizer operating at a sampling rate of $1 \mathrm{MHz}$. Both the PXI 5142 and 5105 are housed in an NI PXI 8108 Embedded Controller. A 50 $\mathrm{mm}$ wide, $50 \mathrm{~mm}$ high and $2.8 \mathrm{~mm}$ thick steel plate is glued on the surface at $(50,0) \mathrm{mm}$ location in range and azimuth and occupies three diagonal pixels, thereby emulating an extended defect. The experimental scene is shown in Fig. 6(a), and the received waveforms are provided in Fig. 6(b). The reconstructed defect images based on the $\mathrm{BP}$ and the proposed approach are shown in Figs. 7(a) and 7(b), respectively. It is observed that the reconstructed result based on BP completely fails, whereas the proposed algorithm correctly acquires the location of the defect while preserving its continuous structure by exploiting both group sparsity and cluster structure.

\section{CONClusion}

In the paper, we focus on imaging of extended defects in thin-walled structures using guided ultrasonic waves in structural health monitoring (SHM) for a multiple-input 


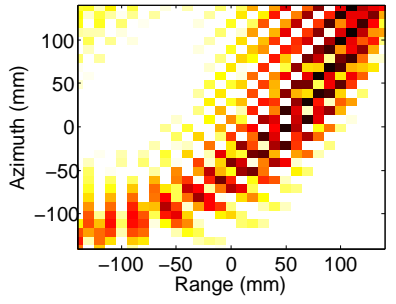

(a)

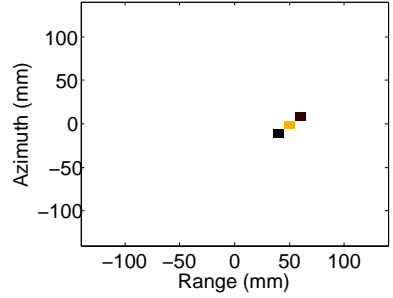

(b)
Fig. 7: Reconstructed result in the experiment. (a) Reconstructed result obtained from BP. (b) Reconstructed result obtained from the proposed algorithm.

multiple-output (MIMO) system. The imaging of defects is formulated as a sparse reconstruction problem. A structureaware Bayesian compressive sensing algorithm was exploited to acquire high-resolution defect images by exploiting both the group sparsity in MIMO systems and cluster structure of the extended defect. Simulation and experimental results demonstrate the superiority of the proposed algorithm over several state-of-the-art approaches.

\section{REFERENCES}

[1] P. C. Chang, A. Flatau, and S. C. Liu, "Health monitoring of civil infrastructure," Structural Health Monitoring, vol. 3, pp. 257-267, 2003.

[2] K. Diamante and D. Soutis, "Structural health monitoring techniques for aircraft composite structures," Progress in Aerospace Sciences, vol. 46, pp. 342-352, 2010.

[3] S. S. Kessler, S. M. Spearing, and C. Soutis, "Structural health monitoring in composite materials using Lamb wave methods," Smart. Mater. Struct., vol. 11, pp. 269-278, 2002.

[4] A. Raghavan and C. E. S. Cesnik, "A review of guided wave structural health monitoring," Shock Vibrations, vol. 39, pp. 91114, 2007.

[5] V. Giurgiutiu and G. Santoni-Bottai, "Structural health monitoring of composite structures with piezoelectric wafer active sensors," AIAA J., vol. 49, pp. 565-581, 2011.

[6] Z. Su, L. Ye, and Y. Lu, "Guided Lamb waves for identification of damage in composite structures: A review," J. Sound and Vibration, vol. 295, pp. 753-780, 2006.

[7] J. Rose, Ultrasonic Waves in Solid Media, Cambridge, U.K.: Cambridge University Press, 1999.

[8] V. T. Rathod and D. R. Mahapatra, "Ultrasonic Lamb wave based monitoring of corrosion type damage in plate using a cir- cular array of piezoelectric transducers," NDT\&E International, vol. 44, pp. 628-636, 2011.

[9] W. J. Staszewski, B. C. Lee, and R. Traynor, "Fatigue crack detection in metallic structures with Lamb waves and 3D Laser vibrometry," Measurement Science and Technology, vol. 18, pp. 727-739, 2007.

[10] Z. Chang and A. Mal, "Scattering of Lamb waves from a rivet hole with edge cracks," Mechanics of Materials, vol. 31, pp. 197-204, 1999.

[11] A. Golato, S. Santhanam, F. Ahmad, and M. G. Amin, "Multimodal sparse reconstruction in Lamb wave-based structural health monitoring," in Proc. SPIE, vol. 9109, Baltimore, MD, May 2014.

[12] G. Konstantinidis, B. W. Drinkwater, and P. D. Wilcox, "The temperature stability of guided wave structural health monitoring systems," Smart Mater. Struct., vol. 15, pp. 967-976, 2006.

[13] C. H. Wang, J. T. Rose, and F. K. Chang, "A synthetic time-reversal imaging method for structural health monitoring," Smart Mater. Struct., vol. 13, pp. 415-423, 2004.

[14] J. Hall and J. Michaels, "Minimum variance ultrasonic imaging applied to an in situ sparse guided wave array,"IEEE Trans. Ultrason., Ferroelectr., Freq. Control, vol. 57, pp. 2311-2323, 2010.

[15] R. M. Levine and R. M. Michaels, "Model-based imaging of damage with Lamb waves via sparse reconstruction," J. Acoust. Soc. Am., vol. 133, pp. 1525-1534, 2013.

[16] R. M. Levine and J. E. Michaels, "Block-sparse reconstruction and imaging for Lamb wave structural health monitoring," IEEE Trans. Ultrason., Ferroelectr., Freq. Control, vol. 61, pp. 10061015, 2014.

[17] Q. Wu, Y. D. Zhang, M. G. Amin, and B. Himed, "Multi-task bayesian compressive sensing exploiting intra-task dependency," IEEE Signal Process. Lett., vol. 22, no. 4, pp. 430-434, 2015.

[18] S. Ji, D. Dunson, and L. Carin, "Multitask compressive sampling," IEEE Trans. Signal Process., vol. 57, no. 1, pp. 92-106, 2009.

[19] Q. Wu, Y. D. Zhang, M. G. Amin, and B. Himed, "Complex multitask Bayesian compressive sensing," in Proc. IEEE ICASSP, Florence, Italy, pp. 3375-3379, May 2014.

[20] L. Yu, H. Sun, J. P. Barbot, and G. Zheng, "Bayesian compressive sensing for cluster structured sparse signals, Signal Process., vol. 92, no. 1, pp. 259-269, 2012.

[21] Q. Wu, Y. D. Zhang, and M. G. Amin, "Continuous structure based Bayesian compressive sensing for sparse reconstruction of time-frequency distributions," in Proc. Int. Conf. Digital Signal Process., Hong Kong, China, pp. 831-836, Aug. 2014.

[22] J. A. Tropp and A. C. Gilbert, "Signal recovery from partial information via orthogonal matching pursuit," IEEE Trans. Info. Theory, vol. 53, no. 12, pp. 4655-4666, 2007. 